\title{
Frühkindliche Karies - was ist diagnostisch und therapeutisch zu beachten?
}

Roswitha Heinrich-Weltzien

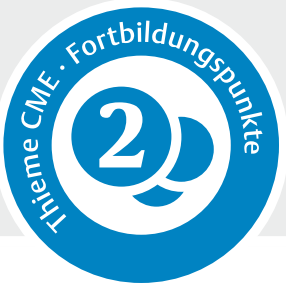

\begin{abstract}
Den aktuellen epidemiologischen Begleituntersuchungen zur Gruppenprophylaxe aus dem Jahre 2017 zufolge, ist die frühkindliche Karies auch in Deutschland ein ungelöstes Gesundheitsproblem [1]. Vor dem Hintergrund der neuen zahnärztlichen Früherkennungsuntersuchungen im System der gesetzlichen Krankenversicherung werden die diagnostischen und therapeutischen Herausforderungen einer präventivorientierten Betreuung von Kleinkindern erörtert.
\end{abstract}

\section{Hintergrund}

In der o.g. repräsentativen bundesweiten Studie wiesen 14\% aller 3-Jährigen kariöse Defekte an durchschnittlich 3,6 Milchzähnen auf, und 5\% der Kinder hatten einen hohen Kariesbefall (dmfs > 4) [1]. Weiterhin waren $74 \%$ aller kariösen Läsionen unversorgt. Akute Zahnschmerzen und daraus resultierende endodontische Folgeerkrankungen sind daher die häufigste Ursache für die Vorstellung von Kleinkindern beim (Kinder)Zahnarzt und deren Krankenhauseinweisungen [2]. Zahnschmerzen gehen darüber hinaus mit Problemen beim Kauen und Schlafen, Appetitlosigkeit, Gewichtsverlust, Verhaltensänderungen, Aktivitätseinschränkungen sowie erheblichen Beeinträchtigungen der Lebensqualität der betroffenen Kinder und ihrer Eltern einher $[3,4]$.

Die Weltorganisation der Zahnärzte (FDI World Dental Federation) forderte daher bereits 2012 in ihrer „Global Caries Initiative“ eine Priorisierung der zahnärztlichen Betreuung auf die Altersgruppe der 0- bis 3-Jährigen sowie die Ausrottung der frühkindlichen Karies bei unter 3-Jährigen [5]. In Deutschland hat der Gesetzgeber mit der Einführung von zahnärztlichen Frühuntersuchungen ab dem ersten Zahn auf das Problem der frühkindlichen Karies reagiert.

\section{Merke}

Seit dem 1. Juli 2019 ist im Rahmen der deutschen vertragszahnärztlichen Regelversorgung eine evidenzbasierte zahnärztliche Betreuung mit dem Fokus auf die Prävention der frühkindlichen Karies gegeben.

\section{Definition}

Die frühkindliche Karies (Early Childhood Caries - ECC) ist eine chronische, multifaktorielle Erkrankung, die von der Amerikanischen Gesellschaft für Kinderzahnheilkunde (American Academy of Pediatric Dentistry - AAPD) als das Vorhandensein eines oder mehrerer kariöser (nicht kavitierter oder kavitierter Läsionen), fehlender Zähne aufgrund von Karies oder gefüllter Milchzahnflächen eines Kindes unter 6 Jahren definiert wird [6].

\section{DEFINITION}

Eine schwere Form der ECC (Severe Early Childhood Caries) liegt vor, wenn

- Zeichen einer Glattflächenkaries bei Kindern jünger als 3 Jahren auftreten,

- im Alter von 3-5 Jahren ein Kariesbefall von $\geq 1$ dmfs vorliegt,

- eine Glattflächenfüllung an oberen Milchfrontzähnen

- und ein Kariesbefall von $\geq 4$ dmfs mit 3 Jahren, von $\geq 5$ dmfs mit 4 Jahren oder von $\geq 6$ dmfs mit 5 Jahren vorliegt [6].

\section{Ätiologie}

Die Ursachen der frühkindlichen Karies sind wie bei jeder anderen Kariesform multifaktoriell, d. h. viele Faktoren interagieren miteinander [6-9]. 


\section{DEFINITION}

Die wichtigsten Risikofaktoren für das Auftreten einer frühkindlichen Karies sind:

- eine frequente Aufnahme zucker- und säurehaltiger Getränke mit der Saugerflasche, insbesondere in der Nacht

- ad libitum Stillen über das 1. Lebensjahr hinaus

- eine unterlassene Zahnpflege bzw. der verspätete Beginn des Zähneputzens mit einer fluoridhaltigen Zahnpaste [6-9].

Die frühe Besiedlung der kindlichen Mundhöhle mit Streptococcus mutans als Folge der Fehlernährung, ein niedriger sozioökonomischer Status und Migrationshintergrund der Familien, ein unzureichendes Gesundheitswissen und -verhalten sowie eine geringe Schulbildung begünstigen weiterhin das Auftreten der frühkindlichen Karies [10].

\section{Klinisches Erscheinungsbild}

Der Kariesbefall im Milchgebiss folgt dem Zahndurchbruch. Daher sind bei der frühkindlichen Karies zuerst die oberen Milchschneidezähne, gefolgt von den ersten Milchmolaren, betroffen. Erste klinische Anzeichen einer frühkindlichen Karies sind initial kariöse Läsionen in Form von weißen opaken Entkalkungen im marginalen Bereich der klinischen Krone ( $\bullet$ Abb. 1 a). Wird eine zahnschädigende Ernährungsweise - Verabreichung hochkalorischer kariogener und/oder erosiver Getränke via Saugerflasche, insbesondere in der Nacht, - fortgeführt und das tägliche Zähneputzen durch die Eltern unterlassen, treten nachfolgend rasch kariöse Läsionen/Kavitationen an den Milcheckzähnen und zweiten Milchmolaren auf ( $\bullet$ Abb. 1 b).
Bei Frühgeborenen können häufiger als bei Reifgeborenen auftretende Schmelzhypoplasien ( $\bullet$ Abb. 2 ) an den Milchzähnen die Kariesentwicklung begünstigen [11], da hypoplastische Zahnareale die Etablierung eines Biofilms auf rauen Zahnoberflächen fördern.

Merke

Eine Schmelzhypoplasie kann durch eine kariöse Läsion maskiert werden.

\section{Leistungsumfang der zahnärztlichen Früherkennungsuntersuchungen}

Den zahlreichen Kampagnen der Zahnärzteschaft mit dem Slogan „Mit dem ersten Zahn zum Zahnarzt“ folgend, stellen immer mehr Eltern ihre Kinder nach dem Durchbruch der ersten Milchzähne beim Zahnarzt nach dem Verweis durch den Kinderarzt oder eigeninitiativ vor. Seit dem 1. Juli 2019 kann der Zahnarzt 3 Früherkennungsuntersuchungen (FU 1a-c) bei Kindern zwischen dem 6 . und 33. Lebensmonat im Rahmen der vertragszahnärztlichen Regelversorgung durchführen ( $\triangleright$ Tab. 1). Weiterhin kann bei allen Kindern eine Fluoridlackapplikation (FLA) zur Schmelzhärtung 2-mal je Kalenderhalbjahr erfolgen. Bis zum vollendeten 72. Lebensmonat haben Kinder wie bisher Anspruch auf die bestehenden 3 vertragszahnärztlichen Früherkennungsuntersuchungen (FU 2).

Der Leistungsumfang der einzelnen FU-Positionen wurde vom Gesetzgeber dezidiert definiert ( $\bullet$ Tab. 1$)$. Praktische Handlungsempfehlungen für den Umgang und die Betreuung von Kleinkindern zur Vermeidung der frühkindlichen Karies im Praxisalltag finden sich in dem online verfügbaren Ratgeber ( $\boldsymbol{A} \mathbf{A b} \mathbf{b}$. $\mathbf{3}$ ), der von der Kassenzahnärztlichen Bundesvereinigung (KZBV) und Bundeszahnärztekammer (BZÄK) herausgegeben wird [12].
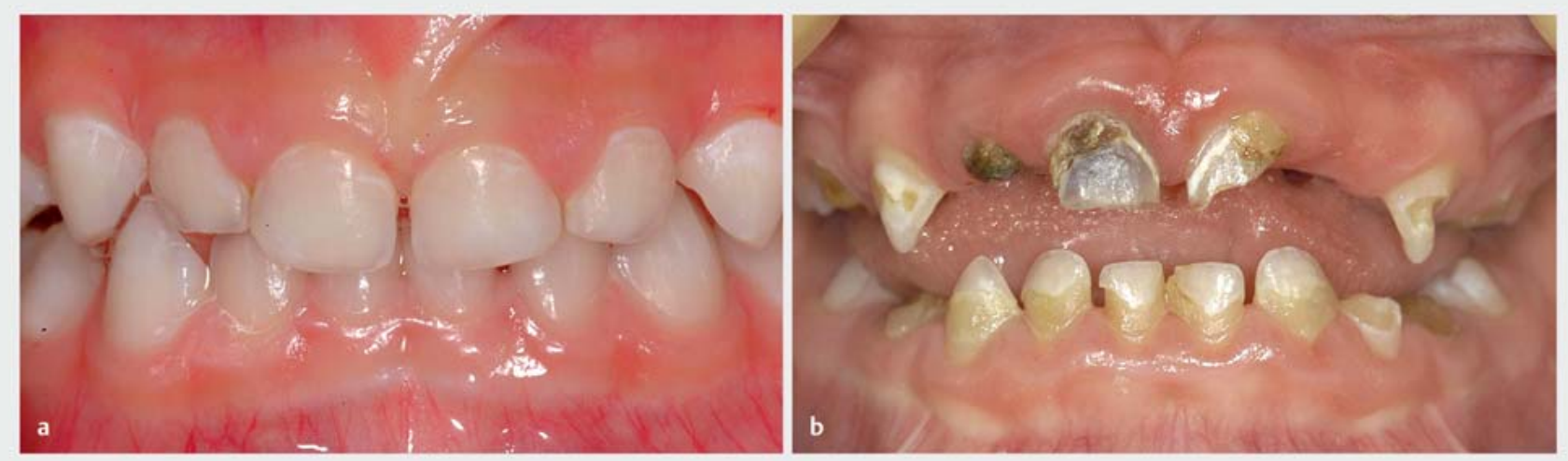

- Abb. 1 a Initial kariöse Läsionen an den oberen Frontzähnen bei einem unter 3-jährigen Patienten. Als Folge der unzureichenden Zahnpflege besteht auch eine plaqueinduzierte Gingivitis im Bereich der Papillen. b Ausgeprägte kariöse Zerstörung aller Zähne bei einem 3-jährigen Patienten. 


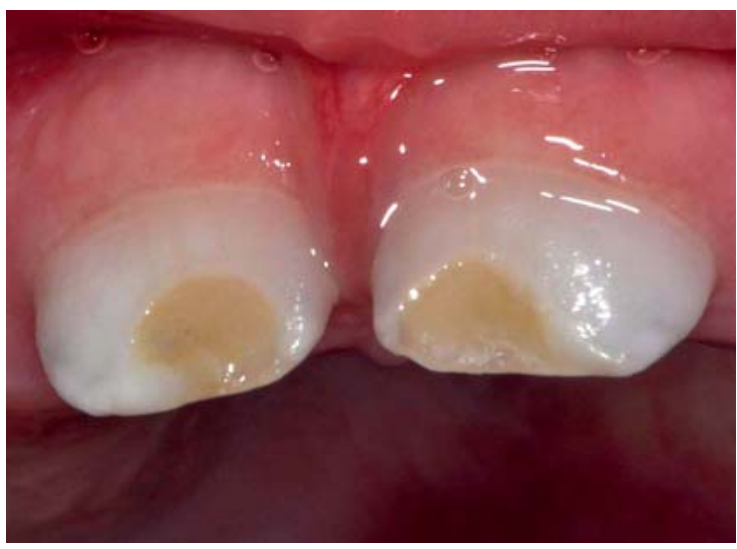

- Abb. 2 Großflächige Hypoplasie an den Zähnen 51 und 61 bei einem 9 Monate alten frühgeborenen Patienten. Auf den hypoplastischen Schmelzarealen hat sich ein Biofilm etabliert, da die Eltern bislang keine Zahnpflege durchführten.

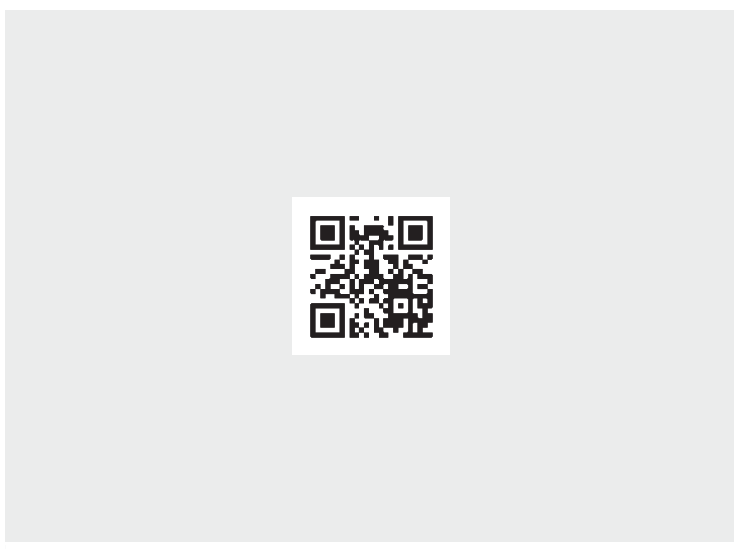

Abb. 3 QR-Code der Titelseite des Ratgebers zur Vermeidung der frühkindlichen Karies, der von der Homepage der Kassenzahnärztlichen Bundesvereinigung und Bundeszahnärztekammer kostenfrei herunterladbar ist.

- Tab. 1 Ärztliche Vorsorgeuntersuchungen und Verweise zu zahnärztlichen Früherkennungsuntersuchungen - seit dem 1. Juli 2019 in Kraft.

\begin{tabular}{|c|c|c|c|}
\hline $\begin{array}{l}\text { Ärztliche Vorsorge- } \\
\text { untersuchung }\end{array}$ & $\begin{array}{l}\text { Verweis durch den } \\
\text { (Kinder)-Arzt }\end{array}$ & $\begin{array}{l}\text { Zahnärztliche Früh- } \\
\text { erkennungsuntersuchung }\end{array}$ & $\begin{array}{l}\text { Leistungen der } \\
\text { Früherkennungsuntersuchung }\end{array}$ \\
\hline $\begin{array}{l}\text { im Zeitraum der U5 } \\
6 .-7 . \text { Lebensmonat }\end{array}$ & $\begin{array}{l}\text { regelhaft zur zahnärzt- } \\
\text { lichen Früherkennungs- } \\
\text { untersuchung }\end{array}$ & $\begin{array}{l}\text { FU 1a und FU Pr } \\
\text { 6.-9. Lebensmonat }\end{array}$ & \multirow{3}{*}{$\begin{array}{l}\text { - Inspektion der Mundhöhle } \\
\text { - Erhebung der Anamnese zum Ernährungs- und } \\
\text { Zahnpflegeverhalten der Betreuungspersonen } \\
\text { - Ernährungs- und Mundhygieneberatung mit dem } \\
\text { Ziel der Keimzahlsenkung durch verminderten } \\
\text { Konsum zuckerhaltiger Speisen und Getränke auch } \\
\text { mittels Nuckelflasche, verbesserte Mundhygiene } \\
\text { durch praktische Anleitung der Betreuungsper- } \\
\text { sonen (FU Pr) zur Mundhygiene beim Kind } \\
\text { - Erhebung der Anamnese zu Fluoridierungsmaß- } \\
\text { nahmen sowie Empfehlung geeigneter Fluoridie- } \\
\text { rungsmittel } \\
\text { - Fluoridlackanwendung zur Zahnschmelzhärtung } \\
\text { (FLA) kann vom 6. bis 72. Lebensmonat 2-mal im } \\
\text { Kalenderhalbjahr nach Beseitigung sichtbarer } \\
\text { weicher Beläge und bei relativer Trockenlegung } \\
\text { abgerechnet werden. }\end{array}$} \\
\hline $\begin{array}{l}\text { im Zeitraum der U6 } \\
\text { 10.-12. Lebensmonat }\end{array}$ & $\begin{array}{l}\text { regelhaft zur zahnärzt- } \\
\text { lichen Früherkennungs- } \\
\text { untersuchung }\end{array}$ & $\begin{array}{l}\text { FU 1b und FU Pr } \\
\text { 10.-20. Lebensmonat }\end{array}$ & \\
\hline $\begin{array}{l}\text { im Zeitraum der U7 } \\
\text { 21.-24. Lebensmonat }\end{array}$ & $\begin{array}{l}\text { regelhaft zur zahnärzt- } \\
\text { lichen Früherkennungs- } \\
\text { untersuchung }\end{array}$ & $\begin{array}{l}\text { FU 1c und FU Pr } \\
\text { 21.-33. Lebensmonat }\end{array}$ & \\
\hline $\begin{array}{l}\text { im Zeitraum der U7a } \\
\text { 34.-36. Lebensmonat }\end{array}$ & $\begin{array}{l}\text { zahnärztliche Früh- } \\
\text { erkennungsuntersuchung }\end{array}$ & \multirow{3}{*}{$\begin{array}{l}\text { ab 34.-72. Lebensmonat } \\
\text { (= 6. Geburtstag) } \\
3 \text { FU } 2 \text { im Mindestabstand } \\
\text { von } 12 \text { Monaten } \\
\text { Kinder von 3, } 4 \text { und } 5 \text { Jah- } \\
\text { ren anstelle der 01-Leistung }\end{array}$} & \multirow{3}{*}{$\begin{array}{l}\text { - Inspektion der Mundhöhle } \\
\text { - Einschätzung des Kariesrisikos anhand des dmfs- } \\
\text { Indexes } \\
\text { - Ernährungs- und Mundhygieneberatung mit dem } \\
\text { Ziel der Keimzahlsenkung durch verminderten } \\
\text { Konsum zuckerhaltiger Speisen und Getränke und } \\
\text { verbesserte Mundhygiene } \\
\text { - Empfehlung geeigneter Fluoridierungsmittel zur } \\
\text { Schmelzhärtung }\end{array}$} \\
\hline $\begin{array}{l}\text { im Zeitraum der U8 } \\
\text { 46.-48. Lebensmonat }\end{array}$ & $\begin{array}{l}\text { zahnärztliche Früh- } \\
\text { erkennungsuntersuchung }\end{array}$ & & \\
\hline $\begin{array}{l}\text { im Zeitraum der U9 } \\
\text { 60.-64. Lebensmonat }\end{array}$ & $\begin{array}{l}\text { zahnärztliche Früh- } \\
\text { erkennungsuntersuchung }\end{array}$ & & \\
\hline
\end{tabular}

\section{Anamnese}

Jeder klinischen Untersuchung des Kleinkindes geht eine sorgfältige Erhebung der Sozialdaten sowie der medizinischen und zahnärztlichen Anamnese voraus. Dabei soll- ten die anamnestischen Fragen vor allem auf die Risikofaktoren der frühkindlichen Karies - Ernährungsweise (Stillen und Flaschengabe), Zahnputzgewohnheiten, Nutzung von Fluoriden - fokussieren. 
Anamnestische Fragen zur Einschätzung des Risikos einer frühkindlichen Karies:

\begin{tabular}{|c|c|}
\hline Fragen & Einschätzung \\
\hline $\begin{array}{l}\text { Hat Ihr Kind eine Grunderkrankung, Behin- } \\
\text { derung, gesundheitliche Beeinträchtigung? }\end{array}$ & $\begin{array}{l}\text { erhöhtes Kariesrisiko bei Gabe hochkalorischer Diäten bei Grunderkrankungen } \\
\text { und Behinderungen }\end{array}$ \\
\hline Nimmt Ihr Kind Medikamente ein? & $\begin{array}{l}\text { erhöhtes Kariesrisiko bei Langzeitgabe von zuckerhaltigen Medikamenten } \\
\text { (Hustensirup, schmerzmittelhaltige Säfte) und säurehaltige Medikamente } \\
\text { (u.a. Asthmasprays) durch Förderung der Schmelzdemineralisation }\end{array}$ \\
\hline $\begin{array}{l}\text { Wird Ihr Kind nachts noch gestillt oder be- } \\
\text { kommt es die Saugerflasche gereicht? }\end{array}$ & $\begin{array}{l}\text { erhöhtes Kariesrisiko bei frequenter nächtlicher Saugerflaschengabe und Stil- } \\
\text { len ad libitum aufgrund der reduzierten nächtlichen Speichelsekretionsrate }\end{array}$ \\
\hline \multirow[t]{2}{*}{$\begin{array}{l}\text { Welches Getränk ist am häufigsten in der } \\
\text { Saugerflasche? }\end{array}$} & $\begin{array}{l}\text { erhöhtes Kariesrisiko in Folge Schmelzdemineralisation bei frequentem Kon- } \\
\text { sum von Früchtetees, zucker- und säurehaltiger Säfte und Schorlen }\end{array}$ \\
\hline & Wasser ist der beste Durstlöscher! \\
\hline $\begin{array}{l}\text { Trinkt Ihr Kind bereits aus der Tasse/Be- } \\
\text { cher? }\end{array}$ & $\begin{array}{l}\text { Im Alter von } 1 \text { Jahr sollte das Kind aus der Tasse/Becher trinken - die Sauger- } \\
\text { flasche wird nicht ad libitum gereicht }\end{array}$ \\
\hline $\begin{array}{l}\text { Putzen Sie Ihrem Kind die Zähne? - wer, } \\
\text { wann, wie oft, womit, wie? }\end{array}$ & erhöhtes Kariesrisiko, wenn Zähne nicht oder nur selten geputzt werden \\
\hline $\begin{array}{l}\text { Welche Kinderzahnpaste mit welchem } \\
\text { Fluoridgehalt verwenden Sie? }\end{array}$ & $\begin{array}{l}\text { Wirksame Kariesprävention ist nur bei Verwendung einer fluoridhaltigen } \\
\text { Zahnpasta mit adäquatem Fluoridgehalt möglich }\end{array}$ \\
\hline $\begin{array}{l}\text { Verwenden Sie andere Fluoridierungsmittel } \\
\text { - Fluoridtabletten, fluoridiertes Speisesalz? }\end{array}$ & $\begin{array}{l}\text { Fluoridtabletten sind nur kariesprotektiv, wenn sie gelutscht werden. Zur Vor- } \\
\text { beugung einer Dentalfluorose muss eine sorgfältige Fluoridanamnese und } \\
\text { Beratung erfolgen }\end{array}$ \\
\hline
\end{tabular}

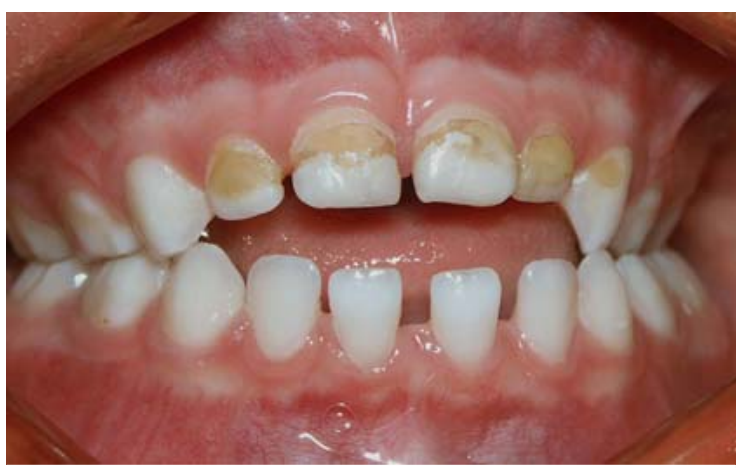

- Abb. 4 Recall eines 3-jährigen Patienten: Im Alter von 1,5 Jahren wurden die offenen Kavitäten nach Handexkavation mit einem Glasionomerzement abgedeckt. Die halbjährliche Fluoridlackapplikation und verbesserte häusliche Mundhygiene führte zur Chronifizierung der zuvor aktiven kariösen Läsionen. Bei verbesserter Compliance wird die Behandlung des offenen Bisses und des seitlichen Kreuzbisses durch den Kieferorthopäden begonnen.

Weiterhin sollten Lutschhabits des Kleinkindes erfragt werden, da diese mit den späteren Konsequenzen eines lutschoffenen Bisses oder seitlichen Kreuzbisses ( $\triangleright$ Abb. 4) einhergehen können.
Zahnärzte, die keinen eigenen Anamnesebogen für Kleinkinder besitzen, finden den Vorschlag eines prophylaxeorientierten Anamnesebogens für das 1.-3. Lebensjahr auf der Homepage der Bundeszahnärztekammer [12].

\section{Zahnärztliche Untersuchung und Diagnostik}

Die Untersuchung des Kleinkindes wird in der Schoß- oder Knie-zu-Knie-Position ( $\boldsymbol{A}$ Abb. 5) empfohlen [12]. In dieser Position öffnet das Kind vielfach reflektorisch den Mund, sodass der Zahnarzt rasch eine orale Inspektion vornehmen kann. Biofilmauflagerungen an den oberen Schneidezähnen sind ein sicherer Indikator für ein hohes Kariesrisiko ( $\bullet$ Abb. 6) und einen zukünftigen Kariesbefall [13]. Da die Bezugsperson, das Kind an den Händen haltend, einen guten Blick in die kindliche Mundhöhle hat, ermöglicht die Entfernung des Biofilms von der Zahnoberfläche mit einer Parodontalsonde oder Watterolle einerseits die sichere Kariesdiagnostik und zeigt andererseits die Notwendigkeit der Zahnreinigung durch die Eltern. Darüber hinaus ist eine differenzierte Kariesdiagnostik von initial kariösen und kavitierten Läsionen nur auf einer sauberen und trockenen Zahnoberfläche möglich. Initial kariöse Läsionen, sog. „White spots“, sind ein deutlicher Indikator für ein hohes Kariesrisiko [14]. Da eine Schmelzhypoplasie auch ein Karies-Risikofaktor sein kann, sollten diese ebenfalls in die Befundung des Zahnarztes einfließen [11]. 


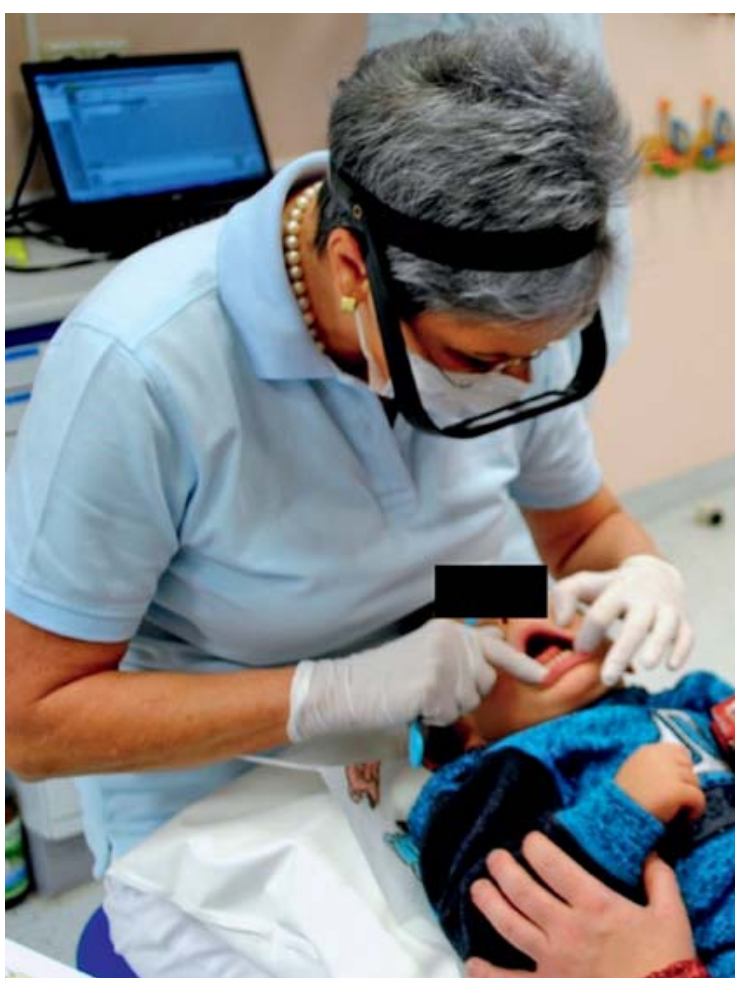

- Abb. 5 Zahnärztliche Früherkennungsuntersuchung eines 2-jährigen Patienten in der Knie-zu-Knie-Position vom Elternteil und Zahnarzt. Das Kind schaut den Elternteil an und erfährt durch das Halten seiner Hände elterliche Zuwendung.

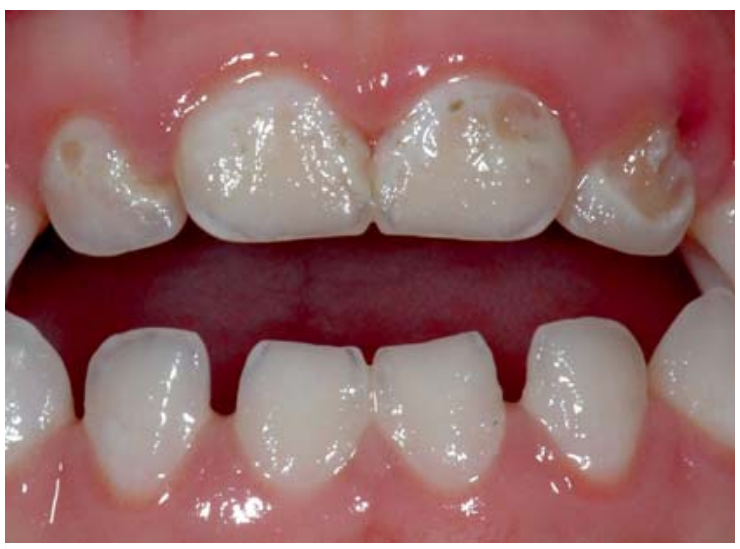

- Abb. 6 Ausgeprägter Biofilm und Gingivitis in der gesamten Frontregion bei einem 3-jährigen Patienten, der auf Verweis des Kinderarztes erstmalig beim Zahnarzt vorgestellt wird. Die Biofilmausdehnung maskiert die initial kariösen und kavitierten Läsionen auf den Labialflächen. Der lutschoffene Biss ist Folge des Daumenlutschens. Das Zähneputzen wurde aufgrund des ablehnenden Verhaltens des Kindes bisher nur sporadisch praktiziert.

\section{Merke}

Initial kariöse Läsionen an den Labialflächen der oberen Milchfrontzähne signalisieren eine unzureichende Mundhygiene und die frequente Gabe zuckerhaltiger Getränke mit der Saugerflasche.

Während sich die Mehrzahl der Kleinkinder problemlos untersuchen lässt, kann im Einzelfall ein Kind mit Weinen, Würgereiz und Ablehnung auf die Mundinspektion reagieren. Wenn diese Reaktionsmuster zuvor mit den Eltern kommuniziert wurden, sollte eine zielführende zahnärztliche Diagnostik dennoch möglich sein.

\section{Ernährungs- und Mundhygieneberatung}

Eine wesentliche Säule der zahnärztlichen Früherkennungsuntersuchung ist das präventive Beratungsgespräch der Eltern, das nachfolgende Inhalte berücksichtigen sollte:

\section{PRAXIS}

Wesentliche Inhalte eines präventiven Gespräches mit der Betreuungsperson

- Stillen ist die beste Ernährungsform des Säuglings. Nach Durchbruch der ersten Zähne fördert nächtliches Stillen ad libitum bei unterlassener Zahnpflege die Kariesentstehung $[6,7,12]$

- Mit Fruchtsäften gefüllte Saugerflaschen sollten weder zum Dauernuckeln noch zum Einschlafen dem Kleinkind gereicht werden [6, 7]

- Sobald das Kind selbstständig sitzen kann und Beikost gegeben wird, sollte auf die Gabe der Saugerflasche verzichtet und am besten Leitungswasser ( $\triangleright$ Abb. 7) mit der Tasse oder einem Becher gereicht werden [15]

- Verzicht auf eine Zuckerzugabe zur Kindernahrung bis zum 2. Geburtstag; Zuckerrestriktion im Kindesalter beugt neben der Karies auch Übergewicht, Adipositas, Diabetes etc. vor [16]

- Keimzahlsenkung kariogener Bakterien durch verringerten Konsum zuckerhaltiger Getränke und Speisen

- Zähneputzen ab dem ersten Zahn mit einer fluoridhaltigen Zahnpaste 2-mal täglich - früh und abends nach der Mahlzeit

- Unterlassene Zahn- und Mundpflege fördern die Kariesentstehung und die Entstehung einer Gingivitis

- Halbjährliche Inanspruchnahme der zahnärztlichen Früherkennungsuntersuchung ermöglicht eine effektive Vorbeugung der frühkindlichen Karies

- Mundgesundheit ist integraler Bestandteil der Allgemeingesundheit des Kindes 


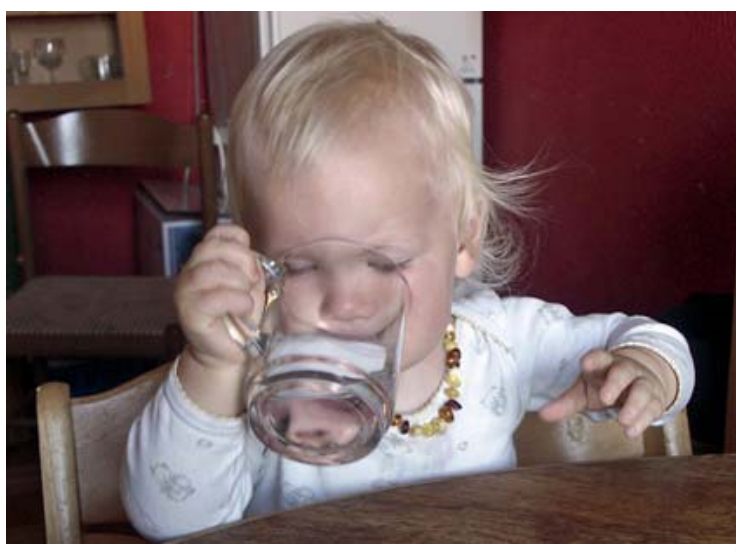

Abb. 7 Zweijähriges Kleinkind beim Wassertrinken aus einer Tasse.

\section{Praktisches Zahnputztraining}

Die angeleitete praktische Durchführung des Zähneputzens durch die Betreuungsperson ist ein wesentlicher Bestandteil der Früherkennungsuntersuchungen (FU Pr). Dass viele Eltern ein solches Training benötigen, zeigte eine videobasierten Studie zum Zähneputzen von Kleinkindern [17]. Die Autoren registrierten eine unzureichende elterliche Zahnputzzeit - 10 Sekunden für die Frontund 13 Sekunden für die Seitenzähne. Sie schlussfolgerten, dass auch bei der Verwendung einer fluoridhaltigen Zahnpaste die Putzzeit und der Fluoridkontakt mit der Zahnoberfläche nicht ausreichen, die frühkindliche Karies zu vermeiden. Neben der Vermittlung der Putzsystematik KAI (Kau-, Außen-Innen-Flächen) sollte gezielt auf die Mundhygienedefizite, die bei der Befundung festgestellt wurden, eingegangen werden. Die Visualisierung des Biofilms (Plaque) durch Anfärbung wird insbesondere bei Kindern mit bereits vorhandenen initial kariösen Läsionen und einer Gingivitis für die Betreuungsperson empfohlen [12]. Weiterhin sollte mit der Demonstration der Lift-theLip-Technik, mit der besonders die am häufigsten kariös betroffenen Oberkieferfrontzähne gut gereinigt werden, Rechnung getragen werden ( $\bullet$ Abb. 8).

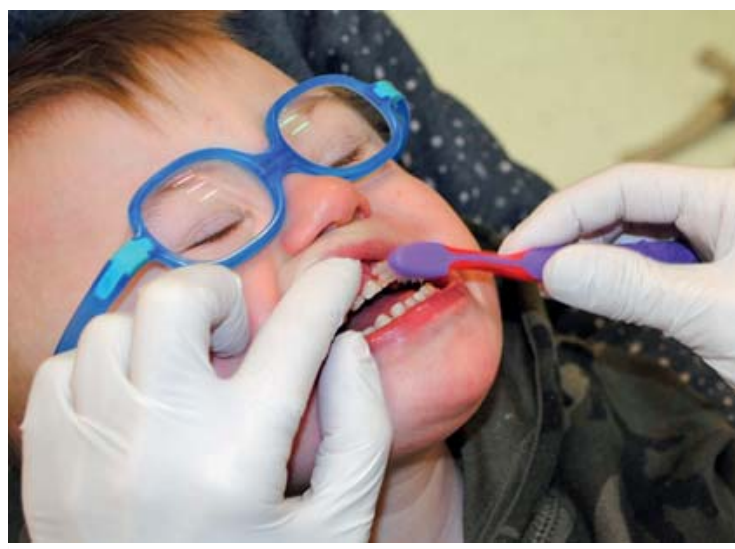

- Abb. 8 Zahnputztraining mit einem Kleinkind - der Mutter des 2-jährigen Patienten wird die Lift-the-LipTechnik demonstriert, die eine gute Reinigung der kariesdisponierten Oberkieferfrontzähne erlaubt.

\section{Merke}

Das praktische Zahnputztraining der Eltern hat das Ziel, eine effiziente Mundhygienepraxis beim Kleinkind zu etablieren. Zähneputzen ist eine Basishygiene, die ritualisiert in die Alltagsroutine eingebettet sein sollte.

\section{Fluoridanwendung - neue Empfehlungen}

Da für die bislang in Deutschland übliche Fluoridkonzentration in Kinderzahnpasten von 500 ppm keine sichere Evidenz einer karieshemmenden Wirksamkeit besteht [18], wurde die Empfehlung zum Fluoridgehalt in Kinderzahnpasten aktualisiert ( Tab. 2) [19]. Mit der Erhöhung der Fluoridkonzentration auf 1000 ppm wird nunmehr eine Zahnpastenmenge für die ersten beiden Lebensjahre von „Reiskorngröße“ empfohlen. Vom 2. bis 6. Geburtstag sollte eine „erbsengroße“ Menge verwandt werden. Bei weiterer Nutzung der 500-ppm-Kinderzahnpaste ist wie zuvor eine „erbsengroße“ Menge in den ersten bei-

- Tab. 2 Aktualisierte Empfehlungen zur Anwendung von Kinderzahnpasten [19].

\begin{tabular}{|l|l|l|l|}
\hline Alter & Konzentration (ppm) & Häufigkeit (täglich) & Menge \\
\hline $\begin{array}{l}\text { ab Durchbruch des 1. Milchzahnes } \\
\text { bis zum 2. Geburtstag }\end{array}$ & 500 & 2-mal & erbsengroß $(0,25 \mathrm{~g})$ \\
\hline & alternativ & & reiskorngroß $(0,125 \mathrm{~g})$ \\
\hline & 1000 & 2 -mal & erbsengroß $(0,25 \mathrm{~g})$ \\
\hline
\end{tabular}




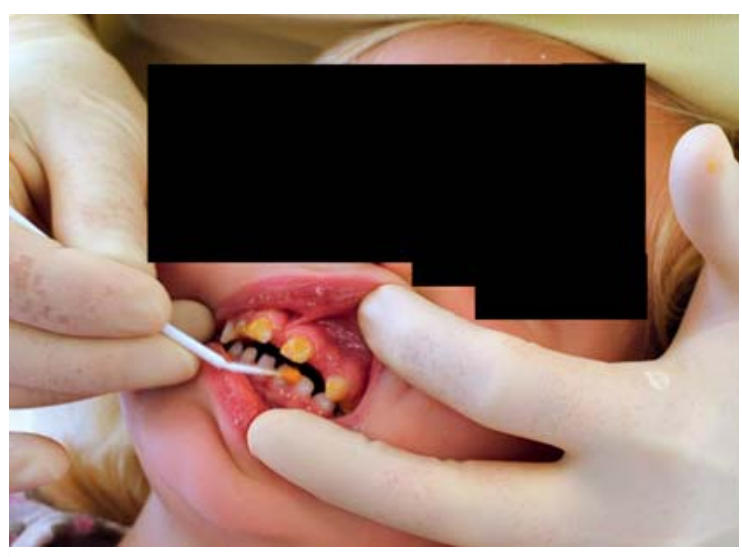

- Abb. 9 Fluoridlackapplikation bei einer 3-jährigen Patientin, die aufgrund der frühkindlichen Karies bereits in Allgemeinanästhesie behandelt wurde.

den Lebensjahren angezeigt. Im Rahmen des Zahnputztrainings mit den Eltern sollte ebenfalls auf die Wahl und die jeweiligen Dosierungsmöglichkeiten der Zahnpaste eingegangen werden.

Merke

Die empfohlene Zahnpastenmenge für die jeweilige Fluoridkonzentration muss eingehalten werden.

Eine weitere Neuerung im Rahmen der Früherkennungsuntersuchung ist die Fluoridlackapplikation, die 2-mal pro Kalenderhalbjahr abgerechnet werden kann. Die Indikation dieser evidenzbasierten kariespräventiven Maßnahme ist beim Vorliegen von initial kariösen Läsionen an den Milchzähnen gegeben. Der Fluoridlack lässt sich beim Kleinkind ebenfalls in der Knie-zu-Knie-Position unkompliziert applizieren ( $\bullet$ Abb.9). Verbunden mit einer guten häuslichen Mundhygiene wird ein weiteres Fortschreiten initial kariöser Läsionen durch ihre Stagnation verhindert ( $\bullet$ Abb. 10). Kleinkinder, die aufgrund einer schweren frühkindlichen Karies in Allgemeinanästhesie behandelt wurden, benötigen als Karies-Risikopatienten ebenfalls eine regelmäßige Fluoridlackapplikation im Rahmen eines engmaschigen Recalls.

Merke

Die Applikation eines Fluoridlackes ist eine evidenzbasierte präventive Therapiemaßnahme zur Chronifizierung bzw. Stagnation initial kariöser Läsionen.

\section{Therapie}

Die zahnärztlichen Früherkennungsuntersuchungen zielen auf eine frühzeitige Diagnostik eines erhöhten Kariesrisikos mit der Erfassung initial kariöser Läsionen, die professionell rein präventiv mit einer Fluoridlackapplikation therapiert werden. Selbst bei bereits eingebrochener Schmelzoberfläche ist eine Stagnation des kariösen Prozesses durch regelmäßige Fluoridlackapplikationen verbunden mit einer effizienten täglichen Zahnpflege möglich ( $\triangleright$ Abb. 10 b) [20]. Liegen kavitierte Läsionen ausschließlich an den oberen Frontzähne vor, empfiehlt sich auch bei sehr kleinen Kindern - zur Vermeidung der Behandlung in Allgemeinanästhesie - die Handexkavation der Karies und der Kavitätenverschluss mit einem Glasionomerzement ( $\bullet$ Abb. 4 ). Bei einem großen Behandlungsbedarf ( $\triangleright$ Abb. 1 ) ist aufgrund der psychoemotionalen Entwicklung und geringen Compliance von Kleinkindern die Behandlung nur in Allgemeinanästhesie
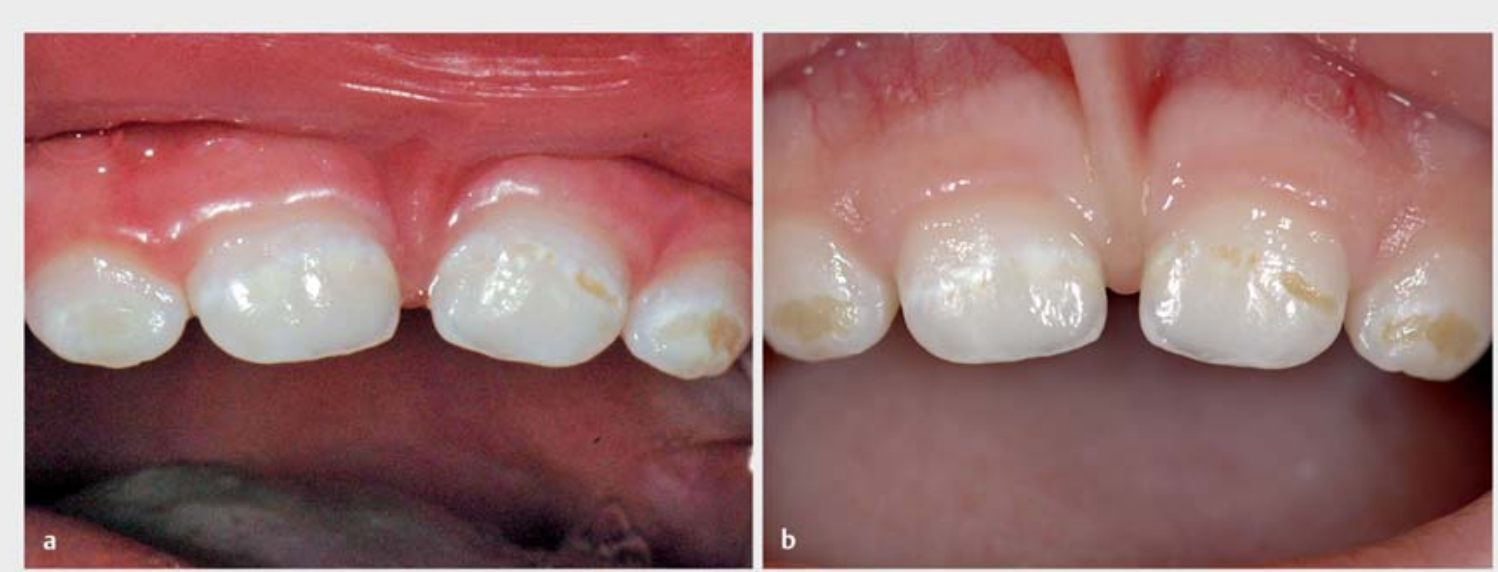

- Abb. 10 a Biofilm und kariöse Läsionen an den oberen Frontzähnen bei einer 18 Monate alten Patientin als Folge des Stillens und unterlassener Zahnpflege. b Die halbjährliche Fluoridlackapplikation und tägliches Zähneputzen mit einer fluoridhaltigen Zahnpaste führte trotz des weiteren Stillens zur Stagnation der kariösen Läsionen, erkennbar an der bräunlichen Verfärbung der eingebrochenen Schmelzoberfläche der Zähne 52, 61, 62. 
möglich. Nicht erhaltungsfähige kariöse Milchzähne müssen extrahiert werden, um die Provokation akuter Exazerbationen und die Entwicklung von Schmelzstrukturstörungen der bleibenden Zähne zu vermeiden [21].

\section{Merke}

Nach der Gebisssanierung in Allgemeinanästhesie müssen Kleinkinder zur Kontrolle des Kariesrisikos in ein präventiv ausgerichtetes Betreuungskonzept einbezogen werden.

\section{Schlussfolgerungen}

Die zahnärztlichen Früherkennungsuntersuchungen zielen auf eine Verbesserung der Zahn- und Mundgesundheit von Klein- und Vorschulkindern im deutschen vertragszahnärztlichen Versorgungskontext ab. Ein präventiv-orientiertes zahnärztliches Betreuungskonzept ab dem ersten Zahn schließt neben der anamnestischen Erhebung der Risikofaktoren für eine frühkindliche Karies die orale Inspektion mit der Erfassung des Biofilmbefalls sowie initial und manifester kariöser Läsionen ein. Dieser sollte sich eine individuelle praktische Unterweisung des Zähneputzens mit einer fluoridhaltigen Zahnpaste der Eltern/Bezugsperson (FU Pr) anschließen. Einen additiven karieshemmenden Effekt hat die halbjährliche professionelle Applikation eines Fluoridlackes; bei vorliegenden initial kariösen Läsionen führt diese zur Chronifizierung oder Stagnation. Invasive therapeutische Maßnahmen können im Zusammenspiel mit einer guten häuslichen Mundhygiene auf diese Weise vermieden werden bzw. auf einen späteren Zeitpunkt mit einer altersbedingten besseren Compliance des Kindes verschoben werden. Um die frühkindliche Karies mit ihren teilweisen schweren Ausprägungen zu vermeiden, bedarf es einer flächendeckenden Umsetzung der neuen Regelungen in der zahnärztlichen Praxis.

\section{KERNAUSAGEN}

- Die frühkindliche Karies ist ein großes Gesundheitsprobleme in der zahnärztlichen Betreuung von Kleinkindern.

- Aufgrund der Einführung der zahnärztlichen Früherkennungsuntersuchungen (FU 1a-1C) in das System der gesetzlichen Krankenversicherung werden zukünftig mehr jüngere als $1 \mathrm{Jahr}$ alte Kinder in der Zahnarztpraxis vorgestellt.

- Anamnestisch sind die Hauptrisikofaktoren für das Auftreten der frühkindlichen Karies - zahnschädigende Ernährungsweise, nächtliches Stillen oder Gebrauch einer Saugerflasche, fehlendes Zähneputzen, fehlende Nutzung einer fluoridhaltigen Zahnpaste - zu erfassen.

- Die Befundung der Kleinkinder schließt die Erfassung des Biofilmbefalls, des Vorkommens von initial kariösen und kavitierten Läsionen ein.

- Die Betreuungspersonen sind umfänglich über Mundhygienemaßnahmen und zahngesunde Ernährungsweisen aufzuklären.

- Mit den Betreuungspersonen ist ein individuelles Zahnputztraining durchzuführen.

- Ab dem Durchbruch des ersten Milchzahnes soll eine fluoridhaltige Kinderzahnpaste entsprechend den aktualisierten Empfehlungen verwandt werden.

- Fluoridlackapplikationen sollen kariesrisikobezogen regelmäßig vorgenommen werden.

\section{Interessenkonflikt}

Die Autorinnen/Autoren geben an, dass kein Interessenkonflikt besteht. 


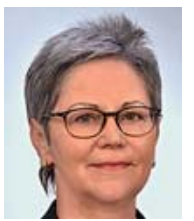

\section{Roswitha Heinrich-Weltzien}

Prof. Dr. med. dent., Jahrgang 1950. 19691974 Studium der Zahnheilkunde an der Friedrich-Schiller-Universität Jena. 19741979 Fachzahnarzt-Ausbildung Kinderzahnheilkunde an der Poliklinik für Präventive Zahnheilkunde und Kinderzahnheilkunde an der Medizinischen Akademie Erfurt. Von 2009 bis 2018 Komm. Direktorin der Poliklinik für Präventive Zahnheilkunde und Kinderzahnheilkunde am Universitätsklinikum Jena. Schwerpunkte: Betreuung von Patienten mit chronischen Erkrankungen und Behinderungen, orale Epidemiologie, Kariesdiagnostik und -prävention, dentale Strukturstörungen.

\section{Korrespondenzadresse}

Prof. Dr. Roswitha Heinrich-Weltzien

Poliklinik für Kieferorthopädie, Sektion Präventive Zahnheilkunde und Kinderzahnheilkunde, Zentrum für Zahn-, Mundund Kieferheilkunde, Universitätsklinikum Jena Bachstraße 18

07740 Jena

roswitha.heinrich-weltzien@med.uni-jena.de

\section{Wissenschaftlich verantwortlich} gemäß Zertifizierungsbestimmungen

Wissenschaftlich verantwortlich gemäß Zertifizierungsbestimmungen für diesen Beitrag ist Prof. Dr. Roswitha Heinrich-Weltzien, Erfurt.

\section{Literatur}

[1] Deutsche Arbeitsgemeinschaft für Jugendzahnpflege e.V. (DAJ). Epidemiologische Begleituntersuchungen zur Gruppenprophylaxe 2016. Bonn: DAJ, 2017. Im Internet: https://www. daj.de/Studien.29.0.html; Stand: 18.05.2020

[2] Bauer T, Otto R, Heinrich-Weltzien R. Gebisszustand von Schmerzpatienten der ersten kinderzahnärztlichen Schwerpunktpraxis in Thüringen im Zeitraum von 2009-2011. Oralprophylaxe Kinderzahnheilkd 2014; 36: 50-56

[3] Corrêa-Faria P, Paixão-Gonçalves S, Paiva SM et al. Dental caries, but not malocclusion or developmental defects, negatively impacts preschoolers' quality of life. Int J Paediatr Dent 2016; 26: 211-219

[4] Martins MT, Sardenberg F, Bendo CB et al. Dental caries remains as the main oral condition with the greatest impact on children's quality of life. PLoS One 2017; 12: e0185365

[5] Fisher J, Johnston S, Hewson N et al. FDI Global Caries Initiative; implementing a paradigm shift in dental practice and the global policy context. Int Dent J 2012; 62: 169-174

[6] American Academy of Pediatric Dentistry (AAPD). Policy on Early Childhood Caries (ECC): Classifications, Consequences, and Preventive Strategies. 2016. Im Internet: http://www. aapd.org/media/Policies_Guidelines/P_ECCClassifications.pdf; Stand: 15.01 .2020
[7] Kassenzahnärztliche Bundesvereinigung (KZBV)/Bundeszahnärztekammer (BZÄK), Hrsg. Frühkindliche Karies vermeiden. Berlin: KZBV/BZÄK, 2014. Im Internet: https://www.kzbv.de/ fruehkindliche-karies-vermeiden.1030.de.html; Stand: 15.01.2020

[8] Leong PM, Gussy MG, Barrow SYL et al. A systematic review of risk factors during first year of life for early childhood caries. Int J Peadiatric Dent 2013; 23: 235-250

[9] Tham R, Bowatte G, Dharmage SC et al. Breastfeeding and risk of dental caries: a systematic review and meta-analysis. Acta Paediatr 2015; 104: 62-84

[10] Harris R, Nicoll AD, Adair PM et al. Risk factors for dental caries in young children: a systematic review of literature. Community Dent Health 2004; 21: 71-85

[11] Schüler IM, Haberstroh S, Dawczynsky K et al. Dental caries and developmental defects of enamel in the primary dentition of preterm infants: case control observational study. Caries Res 2018; 52: 22-31

[12] Kassenzahnärztliche Bundesvereinigung/Bundeszahnärztekammer, Hrsg. Praktischer Ratgeber für die zahnärztliche Praxis - Frühkindliche Karies vermeiden. 2. Aufl. Berlin KZBV/ BZÄK, 2019. Internet: https://www.bzaek.de/fileadmin/PDFs/ b16/ecc-ratgeber.pdf; Stand: 15.01.2020

[13] Alaluusua S, Malmivirta R. Early plaque accumulation - a sign for caries risk in young children. Community Dent Oral Epidemiol 1994; 22: 273-276

[14] André Kramer AC, Skeie MS, Skaare AB et al. Caries increment in primary teeth from 3 to 6 years of age: a longitudinal study in Swedish children. Eur Arch Paediatr Dent 2014; 15: 167173

[15] Koletzko B, Bauer CP, Cierpa M et al. Ernährung und Bewegung von Säuglingen und stillenden Frauen. Aktualisierte Handlungsempfehlungen von „Gesund ins Leben - Netzwerk Junge Familie“. Monatsschr Kinderheilkd 2016; 164: 765-789

[16] World Health Organization (WHO). Guideline: Sugars Intake for Adults and Children. Geneva: WHO, 2015

[17] Zeedyk MS, Longbottom C, Pitts NB. Tooth-brushing practices of parents and toddlers: A study of home-based videotaped sessions. Caries Res 2005; 39: 27-33

[18] Walsh T, Worthington HV, Glenny AM et al. Fluoride toothpastes of different concentrations for preventing dental caries in children and adolescents. Cochrane Database Syst Rev 2010; (1): CD007868. doi:10.1002/14651858.CD007868. pub2

[19] Schiffner U. Neue Empfehlung Fluorid-Kinderzahnpasten. Oralprophylaxe Kinderzahnheilkd 2018; 40: 161

[20] Weintraub JA, Ramos-Gomez F, Jue B et al. Fluoride varnish efficacy in preventing early childhood caries. J Dent Res 2006; 85: $172-176$

[21] Heinrich-Weltzien R, Kühnisch J. Frühkindliche Karies (Early Childhood Caries - ECC) - Folgen der Nichtbehandlung. Quintessenz 2019; 70: 516-524

Bibliografie

DOI https://doi.org/10.1055/a-1072-0627

ZWR - Das Deutsche Zahnärzteblatt 2020; 129: 262-272

(c) Georg Thieme Verlag KG Stuttgart · New York ISSN 0044-166X 


\section{Punkte sammeln auf CME.thieme.de}

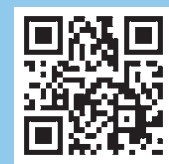

Diese Fortbildungseinheit ist in der Regel 12 Monate online für die Teilnahme verfügbar.

Den genauen Einsendeschluss finden Sie unter https://cme.thieme.de/.

Sollten Sie Fragen zur Online-Teilnahme haben, finden Sie unter https://cme.thieme.de/hilfe

eine ausführliche Anleitung. Wir wünschen viel Erfolg beim Beantworten

der Fragen!

Unter https://cme.thieme.de/CXEASXN oder über den QR-Code kommen Sie direkt zur Startseite des Wissenstests.

\section{Frage 1}

Die frühkindliche Karies ist ein...

A regionales Gesundheitsproblem im Kleinkindalter.

B globales Gesundheitsproblem im Kleinkindalter.

C länderspezifisches Gesundheitsproblem im Kleinkindalter.

D unbedeutendes Gesundheitsproblem im Kleinkindalter.

E Gesundheitsproblem im Kleinkindalter isolierter gesellschaftlicher Gruppen.

\section{Frage 2}

Eine schwere Form der frühkindlichen Karies liegt vor, wenn ...

A eine Karies bei einem Kind unter 6 Jahren auftritt.

B 1 Zahn bei einem Kind unter 6 Jahren kariös bedingt extrahiert wurde.

C 2 Zähne bei einem Kind unter 6 Jahren kariös bedingt extrahiert wurden.

D 1 oberer Milchfrontzahn unter 6 Jahren kariös bedingt extrahiert wurde.

E Zeichen einer Glattflächenkaries bei Kindern jünger als 3 Jahren auftreten.

\section{Frage 3}

Ein Risikofaktor für das Auftreten einer frühkindlichen Karies ist...

A das fehlende Stillen des Kindes.

B die frequente nächtliche Aufnahme zucker- und säurehaltiger Getränke mit der Saugerflasche.

C eine Erkältungskrankheit des Kindes.

D eine sensorische Behinderung des Kindes.

$\mathrm{E}$ ein hoher Sozialstatus der Eltern.

\section{Frage 4}

Die am häufigsten betroffenen Zähne beim Krankheitsbild der frühkindlichen Karies sind...

A die unteren Milchfrontzähne.

B die oberen Milchfrontzähne.

C die oberen zweiten Milchmolaren.

D die unteren zweiten Milchmolaren.

E alle Unterkieferzähne.

\section{Frage 5}

Bei Frühgeborenen wird die Entwicklung einer frühkindlichen Karies begünstigt, wenn...

A ein Engstand der Milchzähne vorliegt.

B ein frühzeitiger Durchbruch der Milchzähne erfolgt.

C Hypoplasien an den Milchzähnen vorliegen.

D Opazitäten an den Milchzähnen vorliegen.

$\mathrm{E}$ ein offener Biss vorliegt.

\section{Frage 6}

Die frühkindliche Karies ist die häufigste Ursache für ...

A eine kieferorthopädische Behandlung.

B eine Hyperaktivität im Kleinkindalter.

C eine Krankenhauseinweisung im Kleinkindalter.

D den Besuch beim Kinderarzt.

E den Besuch beim HNO-Arzt.

- Weitere Fragen auf der folgenden Seite... 


\section{Punkte sammeln auf CME.thieme.de}

Fortsetzung...

\section{Frage 7}

Die frühkindliche Karies beeinflusst nachhaltig ...

A die kieferorthopädische Behandlungsfähigkeit der Kinder.

B die Lebensqualität von Kleinkindern und ihren Eltern.

C die Einstellung der Kinder zu ihrer Mundgesundheit.

D das Verhalten der Betreuungspersonen zur kindlichen Mundgesundheit.

E das Ernährungsverhalten der Kinder.

\section{Frage 8}

Eine wesentliche Säule der zahnärztlichen Früherkennungsuntersuchungen ist...

A die Erfassung von Lutschhabits.

B die Fluoridanamnese.

C die medizinische Anamnese des Kindes.

D die soziale Anamnese der Eltern.

E das praktische individualisierte Zahnputztraining mit der Betreuungsperson.

\section{Frage 9}

Die Applikation eines Fluoridlackes bei Kindern ab dem 6. Lebensmonat ist...

A eine professionelle Maßnahme zur Desensibilisierung des Kleinkindes.

B eine angstreduzierende Behandlungsmaßnahme.

C die Vorbereitung des Kleinkindes auf die zahnärztliche Behandlung.

D eine evidenzbasierte kariesprotektive Maßnahme.

E eine Maßnahme der Verhaltensführung beim Kleinkind.

\section{Frage 10}

Bei Verwendung einer Zahnpaste mit einem Fluoridgehalt von 1000 ppm wird in den ersten beiden Lebensjahren eine Zahnpastenmenge von
A Erbsengröße
B Reiskorngröße
$C$ einem Hauch
D Reiskorn- bis Erbsengröße
E einem breiten Band auf dem Bürstenkopf empfohlen. 